\title{
Concurrent Isolation of Granulocytes and Lymphocytes with Unaltered Permeability, Energy State, and Metabolic Capacity in Vitro
}

\author{
Edward I. Cullen and Fedor Medzihradsky \\ Departments of Biological Chemistry and Pharmacology, The University of Michigan \\ Medical School, Ann Arbor, Michigan 48109
}

Received September 21, 1979

Although numerous procedures have been described for the isolation of either granulocytes or lymphocytes, few focused on the concurrent separation of these cells from the same sample of blood. Those reported (e.g., (1)) are based on the use of discontinuous gradients of Ficoll-Hypaque (sodium diatrizoate), a method introduced by Boyum (2). However, a common disadvantage of these procedures is the substantial contamination of the isolated granulocytes and lymphocytes by erythrocytes and platelets. Our aim, to extend the investigation of the mechanisms of cellular drug transport in leukocytes $(3,4)$ to granulocytes and lymphocytes, required the availability of corresponding cellular fractions of high purity in which the cells fulfill stringent criteria of viability. The presence of an intact plasma membrane during experiments focusing on transport is an essential prerequisite in utilizing cells in such studies. Plasma membrane integrity has to be evaluated considering stringent criteria, sensitive enough to detect even slight perturbations of the plasma membrane (5). Furthermore, the isolated cells have to fulfill the aforesaid and additional criteria of viability over a prolonged time, to allow their use in experiments in vitro. We have previously described a method for the isolation of human and rat leukocytes, erythrocytes, and platelets which fulfilled the above outlined requirements (6). This paper describes a procedure for the concurrent isolation and separation of human granulocytes and lymphocytes, focusing on high purity and long-term viability in vitro.

\section{MATERIALS AND METHODS}

Human blood was obtained by venipuncture from healthy male and female volunteers who were not taking medications. The separation fluid 
"Isolymph", an isotonic solution of Ficoll and sodium diatrizoate, was a product of Gallard-Schlesinger, Carle Place, New York. Clinical grade dextran with an average molecular weight of $2.52 \times 10^{5}$, and crystalline bovine serum albumin were obtained from Sigma Chemical Company, St. Louis, Missouri. The latter company and Boehringer-Mannheim, Indianapolis, Indiana, were sources for the other biochemicals and for enzymes utilized in this work. Hexamethyldisilazane, a siliconizing agent, was purchased from the Anspec Company, Ann Arbor, Michigan. All other chemicals used were of reagent grade.

Buffered media and solutions. Table 1 lists the composition of the three principal buffers used in this work. Two additional media were used. Medium D: $3 \%$ dextran (w/v) and $10 \mathrm{~mm}$ reduced glutathione (GSH) in medium $\mathrm{C}$. The $\mathrm{pH}$ was adjusted to 7.4 with $1 \mathrm{~N} \mathrm{NaOH}$. Medium E: $50 \mathrm{~mm} \mathrm{Na} \mathrm{N}_{2}$ EDTA and $50 \mathrm{mM} \mathrm{Na}{ }_{4}$ EDTA adjusted to $\mathrm{pH} 7.4$ with $1 \mathrm{~N}$ $\mathrm{NaOH}$.

Siliconization of glassware. All glassware, except the Vacutainer tubes, utilized in this work was siliconized by subjecting it to the following procedure. The thoroughly clean and dried glass was filled with a solution of $1 \%$ hexamethyldisilazane in benzene previously warmed to $60^{\circ} \mathrm{C}$. After cooling to room temperature, the siliconizing medium was poured off, and the glassware was allowed to dry in air, followed by heating at $200^{\circ} \mathrm{C}$ for $10 \mathrm{~min}$.

Cell counting. Electronic cell counting was carried out utilizing a Cytograf, Model 6301 (Ortho Instruments, Westwood, Mass.). Differential cell counts on smears treated with Wright's stain were carried out in the Hematology Laboratory, Department of Internal Medicine, The University of Michigan.

Determination of protein. The method of Lowry et al. (7) was utilized, with bovine serum albumin as standard.

Determination of cellular potassium and sodium. Aliquots of the cellular suspension corresponding to approximately $1 \times 10^{7}$ cells were centrifuged in polyethylene tubes at $12,000 \mathrm{~g}$ for $3 \mathrm{~min}$ at $4^{\circ} \mathrm{C}$. The pellet was gently resuspended with ice-cold $0.27 \mathrm{M}$ sucrose, previously neutralized

TABLE 1

Composition and Properties of Buffers

\begin{tabular}{|c|c|c|c|c|c|c|c|}
\hline \multirow[b]{2}{*}{ Medium } & \multicolumn{5}{|c|}{ Concentration (mM) } & \multirow[b]{2}{*}{ pH } & \multirow{2}{*}{$\begin{array}{l}\text { Osmolarity } \\
\text { (mosm/liter) }\end{array}$} \\
\hline & $\mathrm{NaCl}$ & $\mathrm{KCl}$ & $\mathrm{MgSO}_{4}$ & $\mathrm{Na}_{2} \mathrm{HPO}_{4}$ & Glucose & & \\
\hline A & 125 & 5 & - & 16 & 10 & $7.4(\mathrm{HCl})$ & 318 \\
\hline $\mathbf{B}$ & 123 & 5 & 1.5 & 16 & 10 & $7.4(\mathrm{HCl})$ & 318 \\
\hline $\mathrm{C}$ & 110 & 5 & - & 16 & - & $7.4(\mathrm{HCl})$ & 278 \\
\hline
\end{tabular}


to $\mathrm{pH} 7.4$ with Tris base, and centrifuged at $5000 \mathrm{~g}$ for $3 \mathrm{~min}$ at $4^{\circ} \mathrm{C}$. The latter procedure was repeated two additional times and the final washed cellular pellet was digested with $50 \mu \mathrm{l}$ of $70 \% \mathrm{HNO}_{3}$ at $80^{\circ} \mathrm{C}$ for $2 \mathrm{hr}$. After the addition of $5 \mathrm{ml}$ of $15 \mathrm{mM} \mathrm{LiCl}$ as internal standard, the samples were subjected to analysis by flame photometry.

Respiration of cells. The rate of oxygen uptake of approximately $1 \times$ $10^{7}$ cells suspended in $2 \mathrm{ml}$ of medium $\mathrm{B}$ was determined in air, utilizing an oxygen electrode YSI, Model 53 (Yellow Springs, Ohio). The initial rate of oxygen consumption was followed on a strip chart recorder.

Determination of ATP. All steps were carried out at $0-4^{\circ} \mathrm{C}$. Approximately $1 \times 10^{7}$ cells were centrifuged at $12,000 \mathrm{~g}$ for $3 \mathrm{~min}$. After the addition of $400 \mu \mathrm{l}$ of ice-cold $0.6 \mathrm{M} \mathrm{HClO}_{4}$, the pellet was disrupted with a narrow glass rod. The suspension was transferred to a $1-\mathrm{ml}$ all-glass Potter-Elvehjem homogenizer, thoroughly dispersed, and centrifuged at $2000 \mathrm{~g}$ for $15 \mathrm{~min}$. Subsequently, $300 \mu \mathrm{l}$ of the clear supernatant were transferred to fresh 4-ml tubes and the $\mathrm{pH}$ of the solution was adjusted to 6.5 with $2 \mathrm{M} \mathrm{K}_{2} \mathrm{CO}_{3}$. After standing in ice for $20 \mathrm{~min}$ to allow complete precipitation of $\mathrm{KClO}_{4}$, the suspension was centrifuged at $2000 \mathrm{~g}$ for $5 \mathrm{~min}$, and the clear supernatant was stored at $-70^{\circ} \mathrm{C}$ until analysis. ATP was determined by reduced pyridine nucleotide fluorometry (8) utilizing a coupled enzymatic reaction with hexokinase and glucose-6-phosphate dehydrogenase. The final concentrations in the assay were (mM): Tris $\mathrm{HCl}\left(\mathrm{pH} \mathrm{8.0)}, 50 ; \mathrm{MgCl}_{2}, 1.0\right.$; dithiothreitol, 0.5; glucose, 0.1; $\mathrm{NADP}^{+}$, 0.05; glucose-6-phosphate dehydrogenase, $0.2 \mu \mathrm{g} / \mathrm{ml}$; hexokinase, 2 $\mu \mathrm{g} / \mathrm{ml}$.

Isolation of cells. In carrying out the individual lettered steps of the isolation procedure shown in Fig. 1, the following comments should be considered:

(A) Blood was collected either into a 10-ml Vacutainer tube containing $12 \mathrm{mg} \mathrm{Na} \mathrm{NDDTA}_{2}$ (lavender stopper) which had been dissolved in $\mathbf{0 . 2}$ $\mathrm{ml}$ Medium A, or into a 10-ml uncoated Vacutainer tube (pink stopper) containing $0.2 \mathrm{ml}$ of Medium $\mathrm{E}$. Both solutions were injected through the rubber stoppers of unopened Vacutainer tubes with a syringe. Mixing of blood with Medium D was accomplished by repeated gentle inversion of the tubes covered with Parafilm. To facilitate sedimentation, the tubes were left standing at a $45^{\circ}$ angle. All subsequent steps were carried out at room temperature.

(B) If not otherwise noted, all centrifugations were carried out in a swing-out bucket rotor of a table-top centrifuge (Dynac, Clay-Adams). The cellular pellet was resuspended by repeated gentle pipetting utilizing a Pasteur pipet with an enlarged, fire-polished opening.

(C) While $10-\mathrm{ml}$ polypropylene tubes were predominantly utilized 


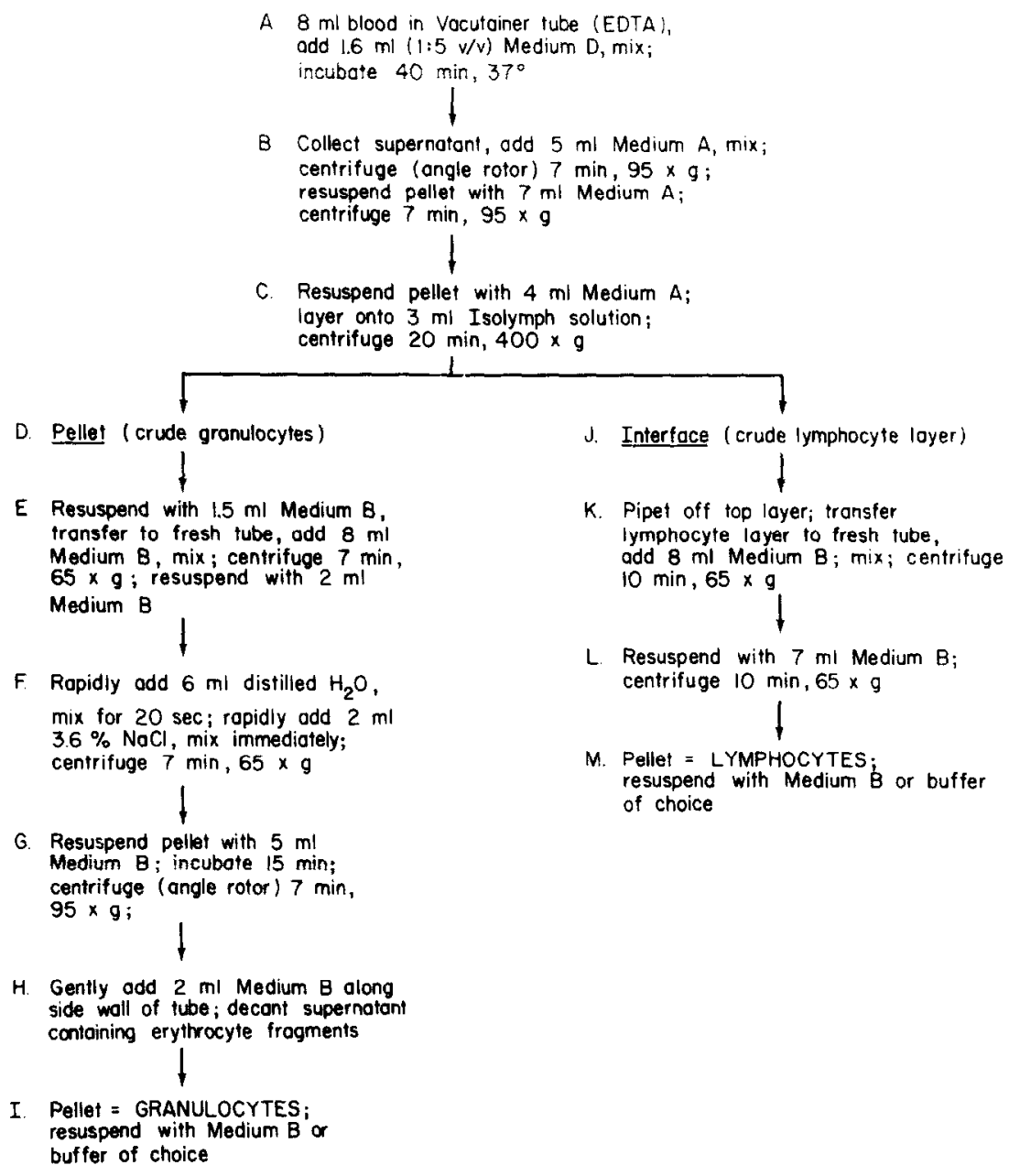

Fic. 1. Scheme for separation of granulocytes and lymphocytes. Additional details pertaining to the individual lettered steps are described under Materials and Methods.

throughout this work, the gradient Medium A-Isolymph was established in a 10-ml polycarbonate tube for better visibility.

(D) The lymphocyte layer and isolymph solution were pipetted off the underlying pellet as completely as possible with a Pasteur pipet.

(E) The transfer of the $1.5-\mathrm{ml}$ cell suspension into a fresh tube containing $8 \mathrm{ml}$ of Medium B serves to dilute out any remaining Isolymph solution and to wash out contaminating platelets. It also avoids contamination of the resuspended granulocyte pellet by other cells adhering to the side wall of the original tube.

(F) The rapid execution of these steps is essential in order to avoid 
hypotonic or hypertonic lysis of granulocytes. To fulfill that requirement, the $\mathrm{H}_{2} \mathrm{O}$ and $\mathrm{NaCl}$ were rapidly delivered along the tube's side wall with syringes, and the tubes were mixed by inversion using a piece of Parafilm as cover.

(G) In this step, an angled centrifuge rotor should be used in order to position the pellet properly for the efficient execution of Step $H$.

(H) This step removes erythrocyte membrane fragments remaining from the hypotonic lysis. By allowing the washing medium to slide along the side wall of the tilted tube, the reddish colored membrane fragments, which loosely accumulated on top of the pellet, were floated off.

(J) Lymphocytes separate as a narrow layer just below the interface between Isolymph and Medium A.

(K) Resuspension of the pellet was carried out by utilizing Pasteur pipets with somewhat enlarged fire-polished openings.

(L) The main purpose of this and the previous washing step (K) was to remove remaining Isolymph solution and residual platelet contamination.

\section{RESULTS}

\section{Isolation and Characteristics of the Cells}

The developed procedure is based on sedimentation, centrifugation, including the use of a simple discontinuous density gradient, and differential hypotonic lysis (Fig. 1). Its requirement for laboratory supplies, biochemicals, and equipment is markedly modest, the latter being limited to a table-top centrifuge and a water bath. Specific recommendations include the Isolymph solution, a mixture of Ficoll and diatrizoate, and siliconization of glassware by the simple procedure described above. The entire isolation can be carried out at room temperature and was routinely completed in $3 \mathrm{hr}$. Of particular significance is the markedly low degree of cross-contamination of either isolated cellular fraction, and the virtual absence of erythrocytes and platelets (Table 2). Contamination by the latter two cell types was a characteristic of the previously described procedures for the concurrent isolation of granulocytes and lymphocytes $(2,9)$. Although no specific efforts were undertaken to further separate the two mononuclear subtypes of leukocytes, the monocyte content of the lymphocyte fraction was markedly low. Only in one isolation, carried out at the early stages of our study, did the monocyte count reach $12 \%$. In five consecutive experiments less than $1 \%$ monocytes were present in the lymphocyte fraction. The ratio of protein to cell count, determined in this study, provides a convenient means of expressing biochemical data. Cellular viability was investigated by several criteria, described in detail below. The property of the cells was assessed immediately after isolation and for up to $2 \mathrm{hr}$ subsequently. It should be noted that at the beginning of 


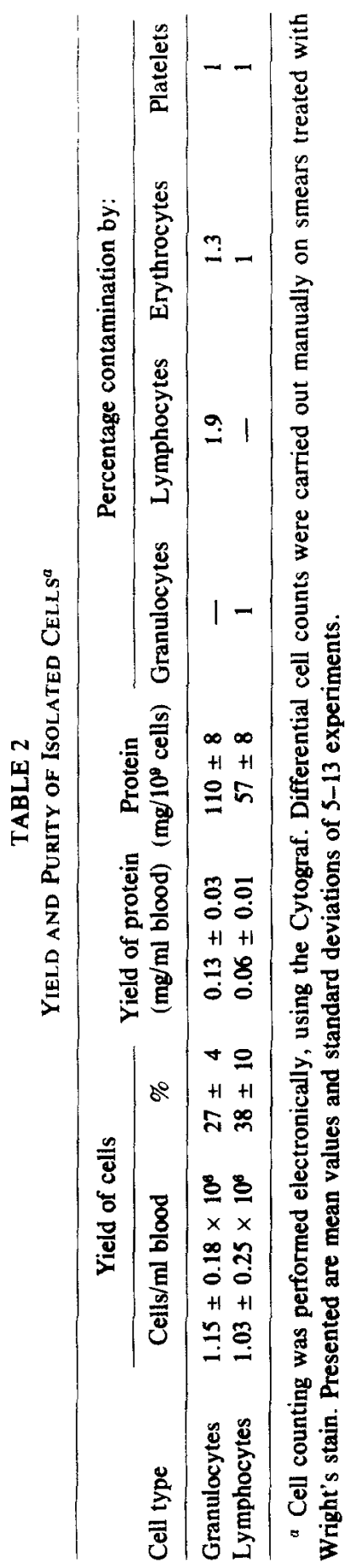


their in vitro evaluation, the isolated granulocytes and lymphocytes represented cells which were removed from their natural environment $2.5-3 \mathrm{hr}$ earlier.

\section{Cell Content of Potassium and Sodium}

The concentration of potassium and sodium in granulocytes and lymphocytes immediately after their isolation was 327 and $82 \mathrm{nEq} / \mathrm{mg}$ protein, and 583 and $100 \mathrm{nEq} / \mathrm{mg}$ protein, respectively (Fig. 2). These values remained constant after a $60-\mathrm{min}$ incubation at room temperature. After $120 \mathrm{~min}$, the sodium content of both cell types was still unchanged whereas the mean cellular potassium concentration showed a slight decline of 7.6 and $8.9 \%$, respectively (Fig. 2).

\section{Cell Content of ATP}

The concentration of this nucleotide in freshly isolated granulocytes and lymphocytes was 12.7 and $24 \mathrm{nmole} / \mathrm{mg}$ protein. These values remained unchanged throughout $2 \mathrm{hr}$ of incubation at room temperature (Fig. 3).

\section{Respiration of Cells}

The rate of oxygen uptake of lymphocytes, measured immediately after their isolation, was markedly higher than that of granulocytes. The respective values were 4.8 and $1.6 \mu \mathrm{l} \mathrm{O}_{2} / \mathrm{mg}$ protein'hr. These values remained essentially constant after $2 \mathrm{hr}$ of in vitro incubation (Fig. 4).

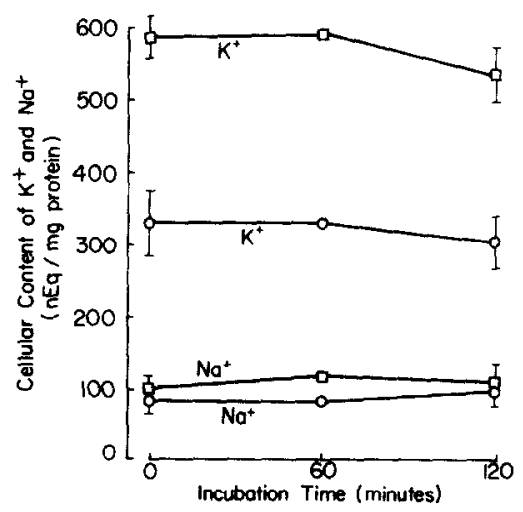

Fig. 2. Content of potassium and sodium in isolated granulocytes $(O)$ and lymphocytes $(\square)$. The cellular ion concentration was determined immediately after isolation and at the indicated times of incubation at room temperature under conditions described under Materials and Methods. Shown are mean values and standard deviations of five experiments, except for the values at $60 \mathrm{~min}$ which represent three experiments. In each experiment cells were isolated from a different sample of blood. 


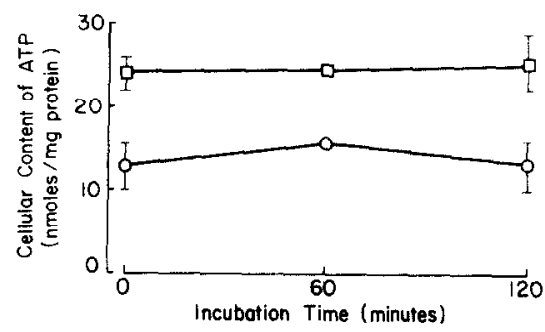

FIG. 3. Content of ATP in isolated granulocytes $(O)$ and lymphocytes $(\square)$. The treatment of cells and the determination of ATP were as described in the legend to Fig. 2 and under Materials and Methods. The results represent mean values and standard deviations of three experiments, except for the values at 60 min which represent two experiments.

\section{DISCUSSION}

The major features of the developed procedure are the concurrent isolation of the two cell types from the same sample of blood, the high purity of the separated granulocytes and lymphocytes, and their long-term in vitro viability, particularly as it relates to unaltered permeability of the plasma membrane. The described procedure requires only simple laboratory supplies and equipment and should thus be routinely applicable in clinical-biochemical laboratories. Although the procedure has been predominantly applied to 8-ml aliquots of blood per tube (Fig. 1), larger volumes can also be used. For example, if starting with $4 \times 8 \mathrm{ml}$ of blood, the combined suspensions obtained in step $C(4 \times 4 \mathrm{ml})$ can be layered in a single tube on top of a 12-ml Isolymph layer. Thorough washing of both the granulocyte pellet and lymphocyte layer by resuspension with medium A is an essential step of the procedure, assuring high purity of the isolated cell fractions. In addition to the washings, differential lysis of contaminating erythrocytes in the granulocyte pellet was employed. In

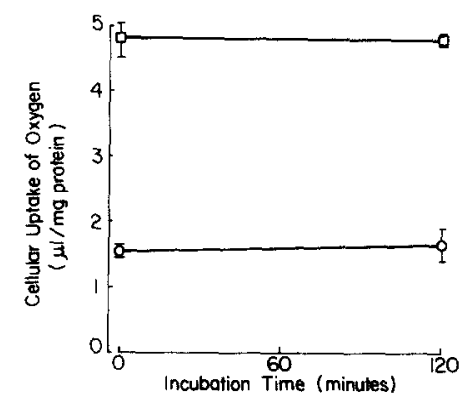

FIG. 4. Uptake of oxygen in isolated granulocytes $(O)$ and lymphocytes $(\square)$. The rate of $\mathrm{O}_{2}$ uptake at $37^{\circ} \mathrm{C}$ was determined for $10 \mathrm{~min}$ immediately after the isolation of cells, and after $2 \mathrm{hr}$ incubation at room temperature. Mean values and standard deviations of three experiments are shown. 
comparison, contamination by erythrocytes in the granulocyte and the lymphocyte fraction separated by a widely used method was 52 and $37 \%$, respectively (9). Furthermore, in the latter procedure the lymphocyte fraction required additional purification for removal of platelet contamination. To ensure unequivocal assessment of the extent of crosscontamination, differential counting of the stained cellular fraction was carried out by hematology staff routinely performing such evaluations. In evaluating the obtained yields of cells, the markedly high purity of both granulocyte and lymphocyte fractions has to be considered. The latter aim was achieved in part by repeated washings and hypotonic lysis, procedures inevitably resulting in decreased yields. In our previously described procedure for the isolation of human leukocytes utilizing Plasmagel (6), the yield of cell isolation was $42 \%$. In the widely applied procedure described by Boyum $(2,9)$ the yields for granulocytes and lymphocytes, both containing substantial contamination by platelets and erythrocytes, were 59 and $97 \%$, respectively. However, as discussed by Dewar (10), these values do not represent recovery of cells from blood but rather reflect the efficiency of separation. After correction, values lower by approximatley $10 \%$ were obtained.

The presence of an intact, nonleaking plasma membrane is a reflection of cell viability and is essential in all studies focusing on transmembrane transport phenomena. The most widely utilized method of assessing cell viability is the exclusion by unperturbed cells of high molecular weight stains, such as trypan blue. However, as we described earlier (5), the latter criterion proved to be an easy test for perturbed cells to pass, i.e., its threshold for indicating membrane damage was markedly high. In that study, trypan blue was still excluded by cells which exhibited marked leakage of potassium and an increased influx of sodium. The cell content of potassium, conveniently determined by flame photometry, proved to be a stringent criterion of even slight perturbations of the plasma membrane of the various cell types investigated (5). Similar findings have been presented by other authors (11). The employment of cellular potassium content as an indicator of membrane perturbation was of value in our efforts to develop a procedure for the isolation of viable blood cells under adverse conditions often prevailing in clinical projects, i.e., delayed separation of cells and/or their isolation after initial removal of plasma (12).

There are only a few reports on the content of potassium and sodium in isolated granulocytes and lymphocytes $(13,14)$. A direct comparison of our data with the published values is difficult due to different references used to express the results. However, the ratio potassium/sodium in granulocytes and lymphocytes isolated separately from different samples of blood were reported to be 3.93 and 3.53 , respectively. Corresponding values in the present study were 3.99 and 5.83 (Fig. 2), indicating that the 
concurrent isolation of these cells did not affect their plasma membrane permeability.

The cellular content of ATP was determined in order to assess the energy status of the isolated cells. It has been shown to decrease as a result of different isolation procedures. A high ratio ATP/ADP was thus interpreted as a criterion for cell viability (15). No data are available on the concentration of ATP in concurrently isolated subtypes of leukocytes. In separately isolated granulocytes and lymphocytes, ATP content was reported to be $10.78 \mathrm{nmole} / \mathrm{mg}$ protein (16) and $17.19 \mathrm{nmole} / \mathrm{mg}$ protein (17), respectively. As in the case of potassium and sodium, our corresponding data of 12.7 and $24.0 \mathrm{nmole} / \mathrm{mg}$ protein compare favorably to those previously reported.

The determination of the respiratory rate of the isolated granulocytes and lymphocytes was included in this study as a measure of their metabolic capacity. Uptake of oxygen has been utilized as a criterion of cell viability in the course of developing procedures for separating subtypes of human leukocytes (18). Oxygen uptake of separately isolated granulocytes and lymphocytes was reported as $2.4 \mu \mathrm{l} \mathrm{O} / \mathrm{mg}$ protein $h \mathrm{hr}$ (19) and $4.6 \mu \mathrm{l} \mathrm{O} / \mathrm{mg}$ protein.hr, respectively $(19,16)$. While the oxygen uptake of lymphocytes in our work correlated well with the above listed data, the corresponding value for granulocytes was lower (Fig. 4). While oxygen consumption of granulocytes, which contain relatively few mitochondria, is expected to be lower than that of lymphocytes, the rate of their respiration can also be affected by the isolation procedure and by the composition of the medium. For example, granulocyte respiration is markedly increased after the addition of autologous serum (16). It is of interest to note that granulocytes in the present study, despite exhibiting a somewhat lower respiratory rate than that previously reported for separately isolated cells, exhibited a higher ATP content than the cells used as reference to compare the oxygen uptake. The latter property of our cells suggests that their respiration was sufficient to maintain a state of high energy.

The favorable characteristics of the concurrently isolated granulocytes and lymphocytes immediately after their isolation were maintained for 2 hr subsequent to their separation which in itself required a 2- to 3-hr period. Thus, the developed procedure yielded cells of marked viability, particularly considering the stringent criteria by which the latter property was assessed in this work. Considering further the virtual lack of crosscontamination in the cellular fractions, the described method for the isolation of granulocytes and lymphocytes, from the same sample of blood, should be of value in studies requiring a high degree of purity and viability, as well as the presence of an intact, nonleaking plasma membrane. 


\section{SUMMARY}

A method has been developed based on sedimentation, discontinuous density gradient centrifugation, and differential lysis for the concurrent isolation from the same sample of blood of granulocytes and lymphocytes with yields of 27 and 38\%, respectively. The isolated cellular fractions were characterized by a lack of cross-contamination and by the virtual absence of erythrocytes and platelets. Plasma membrane integrity, energy status, and metabolic capacity of the isolated cells were assessed by the cell contents of potassium, sodium, ATP, and by the uptake of oxygen. The respective values for these parameters of cell viability compared favorably with previously reported data for separately isolated granulocytes and lymphocytes. The cellular characteristics determined immediately after the isolation of the cells were maintained throughout a 2-hr period at room temperature.

\section{ACKNOWLEDGMENTS}

We would like to thank Mrs. Ruth A. Prieskorn and Merilee H. Valentine for readily provided professional assistance in the collection of blood samples. This work has been supported by USPHS Grant 5 ROI EY02450 and by a stipend (E.I.C.), from the University of Michigan Cancer Research Institute.

\section{REFERENCES}

1. English, D., and Andersen, B. R., J. Immunol. Methods 5, 249 (1974).

2. Boyum, A., Scand. J. Clin. Lab. Invest. 21 (Suppl. 97), 77 (1968).

3. Marks, M. J., and Medzihradsky, F., Mol. Pharmacol. 10, 837 (1974).

4. Marks, M. J., and Medzihradsky, F., Biochem. Pharmacol. 23, 2951 (1974).

5. Medzihradsky, F., and Marks, M. J., Biochem. Med. 13, 164 (1975).

6. Medzihradsky, F., Marks, M. J., and Metcalfe, J. I., Biochem. Med. 10, 153 (1974).

7. Lowry, O. H., Rosebrough, N. J., Farr, A. L., and Randall, R. J., J. Biol. Chem. 193, 265 (1951).

8. Lowry, O. H., Roberts, N. R., and Kapphahn, J. I., J. Biol. Chem. 224, 1047 (1957).

9. Boyum, A., Tissue Antigens 4, 269 (1974).

10. Dewar, C., J. Immunol. Methods 20, 301 (1978).

11. Baur, H., Kasparek, S., and Pfaff, E., Hoppe-Seyler's Z. Physiol. Chem. 356, 827 (1975).

12. Medzihradsky, F., and Metcalfe, J. I., J. Lab. Clin. Med. 85, 342 (1975).

13. Lichtman, M. A., and Weed, R. I., Blood 34, 645 (1969).

14. Segel, G. B., and Lichtman, M. A., J. Cell Physiol. 93, 277 (1977).

15. Frei, J., in "Biological Activity of the Leukocyte" (G. E. W. Wolstenholme and M. O'Connor, Eds.), p. 86. Little, Brown, Boston, 1961.

16. Marchand, J.-C., Leroux, J.-P., and Cartier, P., Eur. J. Biochem. 31, 483 (1972).

17. Pross, S. H., Klein, T. W., and Fishel, C. W., Proc. Soc. Exp. Biol. Med. 154, 508 (1977).

18. Rabinowitz, Y., Blood 23, 811 (1964).

19. Hedeskov, C. J., and Esmann, V., Blood 28, 163 (1966). 